\title{
Geniculocalcarine Tract Disintegration after Ischemic Stroke: A Diffusion Tensor Imaging Study
}

\author{
Y. Zhang, S. Wan, and X. Zhang
}

\begin{abstract}
BACKGROUND AND PURPOSE: Our aim was to investigate the disintegration of the geniculocalcarine tract by using DTI-derived parameters in cases of unilateral occipital or temporal-occipital ischemic stroke with geniculocalcarine tract involvement and to determine whether geniculocalcarine tract fibers affected by infarction and unaffected ipsilateral geniculocalcarine tract fibers have different disintegration processes.
\end{abstract}

MATERIALS AND METHODS: Seventy-one patients underwent routine MR imaging and DTI of the brain. Fractional anisotropy and mean diffusivity of the geniculocalcarine tract fibers affected by infarction, ipsilateral unaffected GCT fibers, and the contralateral geniculocalcarine tract were measured and compared at 5 different time points (from $<1$ week to $>1$ year) poststroke.

RESULTS: The fractional anisotropy of geniculocalcarine tract fibers affected by infarction $(0.27 \pm 0.06)$ was lower than that of contralateral GCT fibers $(0.49 \pm 0.03)$. The fractional anisotropy of geniculocalcarine tract fibers affected by infarction was not different in the first 3 weeks $(P=.306)$. The mean diffusivity of geniculocalcarine tract fibers affected by infarction $(0.53 \pm 0.14)$ was lower than that of the contralateral GCT fibers $(0.79 \pm 0.07)$ in the first week but higher after the second week $(0.95 \pm 0.20$ to $0.79 \pm 0.06)$. The mean diffusivity gradually increased until it was equal to the mean diffusivity of CSF after the eighth week ( $2.43 \pm 0.26)$, at which time both the fractional anisotropy and mean diffusivity values stabilized. The fractional anisotropy $(0.50 \pm 0.04)$ and mean diffusivity $(0.77 \pm 0.06)$ of the ipsilateral unaffected GCT fibers were similar to those of the contralateral GCT fibers $(0.50 \pm 0.03$ and $0.79 \pm 0.07)$ during the first 3 weeks. The fractional anisotropy then gradually decreased (from $0.42 \pm 0.03$ to $0.27 \pm 0.05$ ), while the mean diffusivity increased (from $0.95 \pm 0.09$ to $1.35 \pm 0.11$, though to a lesser degree than in the corresponding geniculocalcarine tract fibers affected by infarction.

CONCLUSIONS: The geniculocalcarine tract fibers affected by infarction and the ipsilateral unaffected GCT fibers showed different disintegration processes. The progressive disintegration of geniculocalcarine tract fibers affected by infarction was stable until the eighth week poststroke. The ipsilateral unaffected GCT fibers began to disintegrate at the fourth week, but to a lesser degree than the geniculocalcarine tract fibers affected by infarction.

ABBREVIATIONS: $\mathrm{GCT}=$ geniculocalcarine tract; $\mathrm{CGCT}=$ contralateral $\mathrm{GCT}$ fibers; $\mathrm{FA}=$ fractional anisotropy; $\mathrm{MD}=$ mean diffusivity; UGCT $=$ ipsilateral unaffected GCT fibers

$\mathbf{S}$ tructural imaging studies have identified the size and location $^{1-6}$ of lesions due to ischemic stroke in conjunction with DTI. ${ }^{7}$ DTI-derived measures are valid markers of ischemic pyra-

Received August 1, 2012; accepted after revision January 31, 2013.

From the Zhongshan Ophthalmic Center (Y.Z.), State Key Laboratory of Ophthalmology, Sun Yat-sen University, Guangzhou, GuangDong, China; Department of Radiology (S.W.), Lu Shan Sanatorium, Nanjing Military Region, Jiu Jiang, Jiang Xi, China; Department of Radiology (X.Z.), Nan Fang Hospital, Southern Medical University, Guangzhou, GuangDong, China; and Department of Radiology (X.Z.), Kang Huang Hospital, Dongguan, GuangDong, China.

This work was supported by the Fundamental Research Funds of the State Key Laboratory of Ophthalmology.

Please address correspondence to Xuelin Zhang, MD, Department of Radiology, Nan Fang Hospital, Southern Medical University, Guangzhou, GuangDong, China, 510515; E-mail: zxlnfyy@163.com

http://dx.doi.org/10.3174/ajnr.A3535 midal tract damage. ${ }^{8}$ The degree of functional motor deficit after a stroke is highly dependent on the overlap of lesions with the corticospinal tract. ${ }^{9}$ DTI studies also have established the relationship between motor impairment in acute phases and damage to the descending motor tracts. ${ }^{10-15}$ The integrity of the pyramidal tract and all of its descending tracts accounts for recovery after stroke. ${ }^{8}$ However, research has primarily concentrated on the pyramidal tract. ${ }^{16-18}$ Although a case describing vision recovery after a perinatal stroke as evidenced by functional and diffusion MR imaging has been reported, ${ }^{19}$ there are no data regarding disintegration of the GCT after an infarction..$^{20-23}$ Understanding ischemic damage to the GCT and probable plasticity is important in determining the prognosis of vision impairment and recovery.

We used DTI-derived parameters to measure the GCT in 71 patients with unilateral occipital or temporal-occipital ischemic 
stroke with GCT involvement. We evaluated the time course of GCT disintegration and hypothesized that GCT fibers affected by infarction and ipsilateral unaffected GCT fibers have different disintegration processes.

\section{MATERIALS AND METHODS \\ Subjects}

The study group consisted of 71 patients (41 men and 30 women; right-handed; age range, 39-89 years; mean age, 63.3 years). Only 1 MR imaging examination was performed in each patient. The time of MR imaging evaluation poststroke was 1 week in 26 patients, $2-3$ weeks in 7 patients, $4-8$ weeks in 7 patients, 8 weeks to 1 year in 15 patients, and $>1$ year in 16 patients. Inclusion criteria were the following: 1) occurrence of the first unilateral occipital or temporal-occipital ischemic stroke; 2) a single infarction lesion that involved the ipsilateral GCT in T1WI, T2WI, and FLAIR sequences; 3) no previous or subsequent cerebral ischemia or hemorrhage; 4) standard physical and occupational therapy poststroke; and 5) absence of other diseases affecting the nervous system.

The research protocol was approved by the Human Ethics Committee for Clinical Research at Southern Medical University (Guangzhou, China). All of the procedures involving the participants were conducted following the Declaration of Helsinki and institutional guidelines in compliance with the stated regulations. Oral and written informed consent was obtained from all participants.

\section{Data Acquisition}

MRI was performed by using a $1.5 \mathrm{~T}$ scanner (Signa Twin; GE Healthcare, Milwaukee, Wisconsin) with an 8-channel phased array head coil. Magnetic field gradients permit up to $40-\mathrm{mT} / \mathrm{m} / \mathrm{s}$ amplitude and a magnetic field gradient switching rate of up to $120 \mathrm{mT} / \mathrm{m} / \mathrm{s}$. The baseline scan was in the axial plane by using a standard head coil. Before and during scanning, head movement was limited by vacuum fixation cushions. All subjects underwent routine brain MR imaging, which included T1WI, T2WI, and FLAIR sequences. Consecutive sections in identical locations for all sequences were acquired with a section thickness of $5 \mathrm{~mm}$. The parameters of T1WI FLAIR included TR/TI/TE, 2500/750/11.9 ms; section thickness, $5 \mathrm{~mm}$; intersection separation, $1.5 \mathrm{~mm}$; matrix size, $320 \times 256$; FOV , $240 \times 180 \mathrm{~mm}$; and double collections. The parameters of T2WI included TR/TE, 4900/99.3 ms; section thickness, $5 \mathrm{~mm}$; intersection separation, $1.5 \mathrm{~mm}$; matrix size, $320 \times 224$; FOV , $240 \times 180 \mathrm{~mm}$; and double collections. The parameters of T2WI FLAIR included TR/TE, 8500/128 ms; section thickness, $5 \mathrm{~mm}$; intersection separation, $1.5 \mathrm{~mm}$; matrix size, $320 \times 192$; FOV, $240 \times 240 \mathrm{~mm}$; and single collection.

DTI was performed in the axial plane by using a spin-echo EPI diffusion tensor sequence after routine MR imaging (TR/TE, 6000/60.1 ms; FOV, $240 \times 240 \mathrm{~mm} ; b=1000 \mathrm{~s} / \mathrm{mm}^{2}$; NEX, 2; diffusion-sensitive gradient direction, 13 ; matrix size, $128 \times 128$; section thickness, $3.5 \mathrm{~mm}$; intersection separation, $0 \mathrm{~mm}$ ).

\section{Data Analysis}

DTI datasets were processed by using Volume One 1.72 (GE Healthcare) and diffusion tensor Visualizer 1.72 software (Tokyo
University, Tokyo, Japan) to obtain directionally encoded color, FA, and ADC images.

The GCT is a fiber tract from the lateral geniculate body, or the subcortex visual center, to the calcarine fissure of the occipital lobe. The afferent nerve fibers of the anterior visual pathway (optic nerve and optic tract) terminate at the dorsal lateral geniculate body, which is the nucleus of the lateral geniculate body ganglion cells, and transform neurons to form the GCT. The GCT passes through the inferior back of the internal capsule and the lenticular nucleus, along the lateral sagittal plane adjacent to the lateral ventricle and is shaped like a convex lamina. ${ }^{24,25}$ The GCT contains the anterior visual pathway afferent fibers and efferent nerve fibers from the occipital lobe to the lateral geniculate body, thalamus, and oculomotor nucleus. ${ }^{26,27}$

ROIs were drawn on the axial directionally encoded color images. The lateral ventricle triangular-region planes (Fig $1 A$, yellow triangle) in which the GCT fibers were completely displayed as green fiber tracts adjacent to the lateral ventricles were selected. The infarction lesions were defined in the same planes of the T1WI and T2WI (Fig 1A, $-C,-E,-G$, brown masses). The ROIs on GCT fibers affected by infarction consisted of GCT fibers overlapped by the infarction lesion (Fig $1 B,-D,-F,-H$, white arrows). The unaffected GCT ROIs consisted of ipsilateral GCT fibers outside the infarction lesion (Fig $1 B,-D,-F,-H$, white triangles). The contralateral GCT ROIs were defined as the entire GCT in the contralateral unaffected hemisphere (Fig $1 B$, yellow circle). The FA and MD of the ROIs were calculated in 3 continuous planes and 3 different regions in each plane by the same observer to obtain a mean value. Calculations were made independently by 3 different observers to obtain the mean value.

\section{Statistical Analysis}

One-way ANOVA was used to evaluate the differences between the FA and MD of the GCT fibers affected by infarction, UGCT, and CGCT on the basis of time points after stroke. The leastsignificant-difference $t$ test was used to compare the FA and MD between 2 different time points poststroke in GCT fibers affected by infarction, UGCT, and CGCT. $P<.05$ was used to determine statistical significance. All analyses were performed by using the Statistical Package for the Social Sciences software, Version 11.0 (SPSS, Chicago, Illinois).

\section{RESULTS}

In the GCT fibers affected by infarction, the FA $(0.27 \pm 0.06)$ was lower than the FA in the CGCT $(0.49 \pm 0.03)$ after the first week poststroke (Table and Fig 2). The FA within the first week (0.27 \pm $0.06)$ was not different from the FA in the second-to-third weeks $(0.29 \pm 0.05, P=.306)$. The FA values stabilized at 8 weeks to 1 year $(P=.643)$. The FA values were significantly different between the different time points poststroke $(F=62.152, P=.000)$. The MD of the GCT fibers affected by infarction $(0.53 \pm 0.14)$ was lower than the MD of the CGCT $(0.79 \pm 0.07)$ in the first week poststroke but was higher after the second week $(0.95 \pm 0.20$ and $0.79 \pm 0.06$, respectively). The MD gradually increased $(2.43 \pm$ $0.26)$ until it was equal to the MD in the CSF after the eighth week $(P=.643)$. The MD stabilized at 8 weeks to 1 year $(P=.087)$. The 

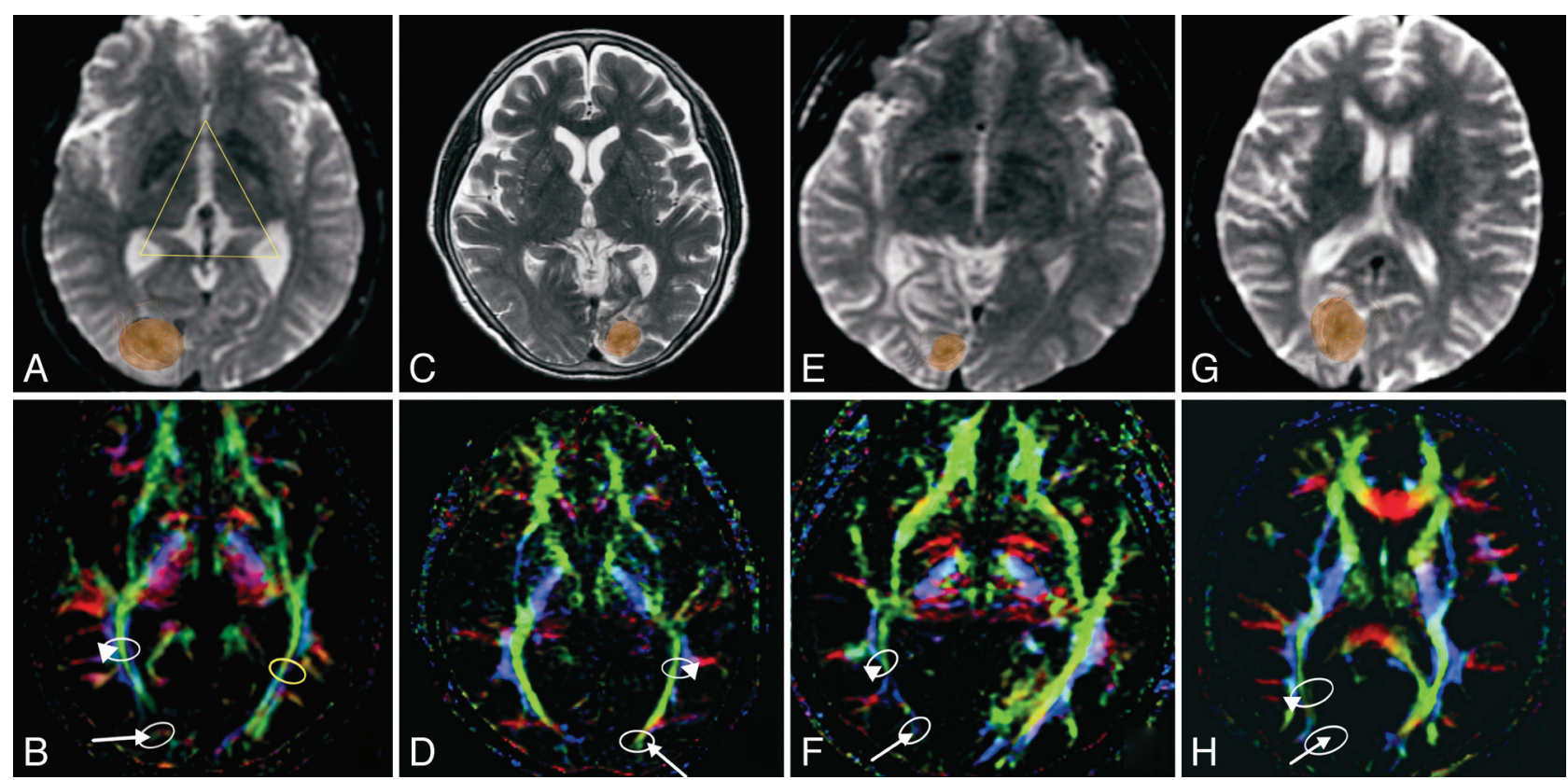

FIG 1. $A$ and $B, A$ 52-year-old man, 16 days postinfarction in the right occipital lobe. The infarcted GCT was hypointense (arrow), while the ipsilateral unaffected GCT (triangle) remained normal. $C$ and D, A 68-year-old man, 6 weeks after infarction in the left occipital lobe. The infarcted GCT was hypointense (arrow), while the signal intensity of the unaffected GCT (triangle) decreased minimally. E and F, A 52-year-old man, 8 months after infarction in the right occipital lobe. The infarcted GCT (arrow) has vanished, while the ipsilateral unaffected GCT (triangle) is significantly hypointense. $G$ and H, A 67-year-old woman, 5 years after infarction in the right occipital lobe. The infarcted GCT (arrow) has vanished. The ipsilateral unaffected GCT (triangle) is remarkably hypointense but complete.

Mean FA and MD values of GCT fibers affected by infarction, UGCT, and CGCT at different times after stroke

\begin{tabular}{|c|c|c|c|c|c|c|c|}
\hline \multirow{2}{*}{$\begin{array}{c}\text { Time after } \\
\text { Stroke }\end{array}$} & \multirow[b]{2}{*}{ No. } & \multicolumn{2}{|c|}{ Infarcted GCT Fibers } & \multicolumn{2}{|c|}{ Unaffected GCT Fibers } & \multicolumn{2}{|c|}{ Contralateral GCT Fibers } \\
\hline & & FA & MD & FA & MD & FA & MD \\
\hline$<1$ Week & 26 & $0.27 \pm 0.06$ & $0.53 \pm 0.14$ & $0.50 \pm 0.03$ & $0.78 \pm 0.08$ & $0.49 \pm 0.03$ & $0.79 \pm 0.07$ \\
\hline 2 3 Weeks & 7 & $0.29 \pm 0.05$ & $0.95 \pm 0.20$ & $0.49 \pm 0.05$ & $0.76 \pm 0.04$ & $0.50 \pm 0.02$ & $0.79 \pm 0.06$ \\
\hline 4 8 Weeks & 7 & $0.18 \pm 0.07$ & $1.88 \pm 0.35$ & $0.42 \pm 0.03$ & $0.95 \pm 0.09$ & $0.51 \pm 0.02$ & $0.77 \pm 0.03$ \\
\hline 8 Weeks 1 year & 15 & $0.08 \pm 0.04$ & $2.43 \pm 0.26$ & $0.31 \pm 0.04$ & $1.07 \pm 0.11$ & $0.49 \pm 0.03$ & $0.78 \pm 0.08$ \\
\hline$>1$ Year & 16 & $0.08 \pm 0.02$ & $2.57 \pm 0.24$ & $0.27 \pm 0.05$ & $1.35 \pm 0.11$ & $0.49 \pm 0.04$ & $0.78 \pm 0.09$ \\
\hline F value & & 62.152 & 293.441 & 134.550 & 101.004 & 1.115 & 0.2222 \\
\hline$P$ value & & .000 & .000 & .000 & .000 & .357 & .925 \\
\hline
\end{tabular}

$\mathrm{a} \times 10^{-3} \mathrm{~mm}^{2} / \mathrm{s}$.

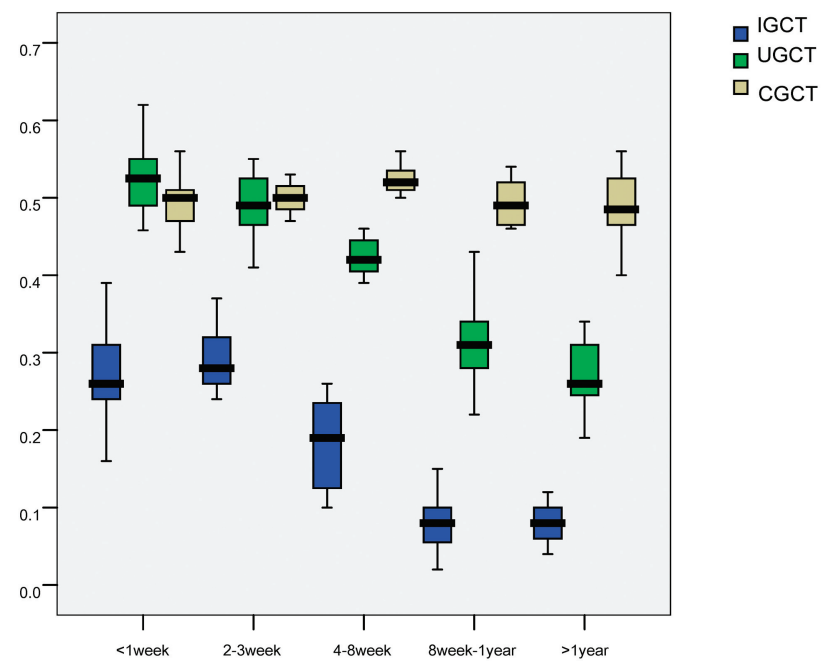

FIG 2. FA values of GCT fibers affected by infarction, UGCT, and CGCT at different times after stroke $\left(\times 10^{-3} \mathrm{~mm}^{2 / \mathrm{s}}\right)$.
MD values were significantly different at the different time points poststroke $(F=293.441, P=.000)$.

In UGCT, the FA $(0.50 \pm 0.04)$ and $\mathrm{MD}(0.77 \pm 0.06)$ were similar to the values in the CGCT $(0.50 \pm 0.03$ and $0.79 \pm 0.07$, respectively) during the first 3 weeks poststroke. From the fourth week, the FA gradually decreased (from $0.42 \pm 0.03$ to $0.27 \pm$ 0.05 ), while the MD gradually increased (from $0.95 \pm 0.09$ to $1.35 \pm 0.11$ ) (Table and Fig 3). However, the ranges were lower compared with the GCT fibers affected by infarction at the corresponding time points. The FA and MD of the UGCT stabilized during the first 3 weeks $(P=.781, P=.686$, respectively). There were significant differences between the values at the different time points poststroke $(F=134.550, P=.000 ; F=101.004, P=$ $.000)$.

In the CGCT, there were no differences between the FA and MD values at the various time points poststroke $(F=1.115, P=$ $.357 ; F=0.222, P=.925$, respectively).

In the first 3 weeks poststroke, the GCT fibers affected by infarction were significantly hypointense, while the UGCT had a 


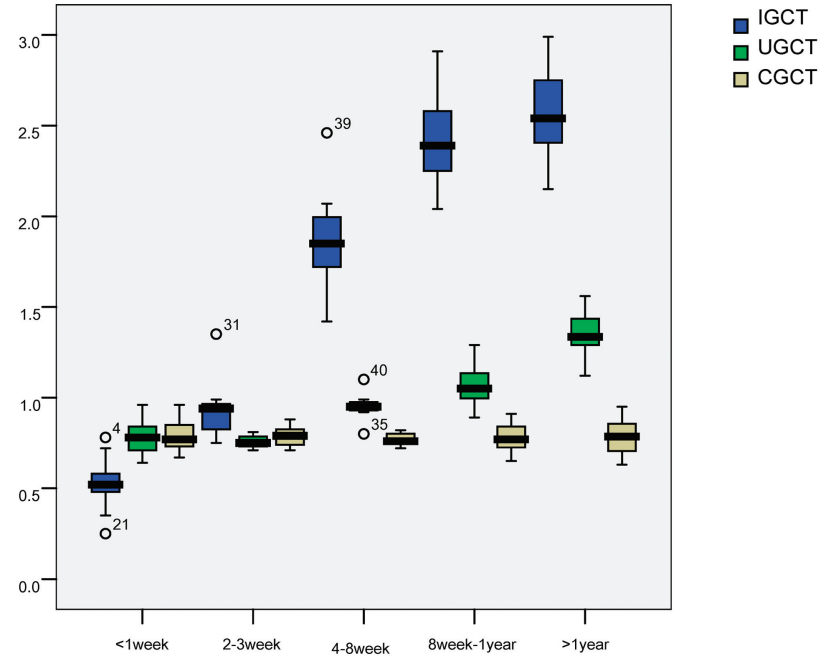

FIG 3. MD values of GCT fibers affected by infarction, UGCT, and CGCT at different times after stroke $\left(\times 10^{-3} \mathrm{~mm}^{2} / \mathrm{s}\right)$.

normal signal intensity and morphology (Fig $1 A,-B)$. During the fourth-to-eighth weeks poststroke, the signal intensity of the UGCT gradually decreased, though their integrity remained intact (Fig $1 C,-D$ ). From the eighth week to 1 year poststroke, the GCT fibers affected by infarction had nearly disappeared, while the UGCT were significantly hypointense (Fig $1 E,-F$ ). The GCT fibers affected by infarction were absent after 1 year poststroke. The UGCT were remarkably hypointense but maintained their complete forms (Fig $1 G,-H$ ).

\section{DISCUSSION}

The FA of the GCT fibers affected by infarction was lower than that of the CGCT immediately after stroke onset (Table). Cells in the central infarcted area where blood flow is dramatically decreased die rapidly after ischemic occlusion. A series of delayed pathophysiologic reactions, including the ischemic cascade, reactions to inflammation, capillary dysfunction, blood-brain barrier damage, and ischemic reperfusion injuries cause necrosis and apoptosis of local neurons and astrocytes. ${ }^{28}$ Degeneration initiates at the end of the distal axon and extends toward the neuron. The flow of information and substances terminates when the neuron is damaged. This phenomenon is known as Wallerian degeneration. ${ }^{23}$ The GCT fibers in the infarction lesion develop Wallerian degeneration. Their axons degenerate and demyelinate at the same time. Reduced FA values in the GCT fibers affected by infarction suggest alterations in axonal attenuation and arrangement. The ordered axonal arrangements and the integrity of the myelin sheath are damaged in GCT fibers affected by infarction. With the disintegration of these barriers, the water molecules in the fiber tracts lose their directivity parallel to the fiber tract and present as a chaotic state. ${ }^{17}$ The FA values, which are significantly lower than the FA values of the CGCT, did not change during the first 3 weeks poststroke. This period may be an important phase of fiber disintegration and a critical time for intervention. The FA stabilized at 8 weeks to 1 year, suggesting that degeneration of the fibers ceased approximately 2 months after infarction.

During the first week poststroke, the GCT fibers affected by infarction had lower MD values than the CGCT. The MD reflects changes in restrictive barriers of the fiber tracts, such as the axonal membrane, myelin sheath, and extracellular space. ${ }^{29,30}$ In the early stages of ischemic stroke, metabolic disorders in the cell induce an increase in intracellular osmotic pressure. Extracellular water enters the cell and causes edema. The volume of the cells increases, while the extracellular spaces decrease. The diffusion resistance increases, and the MD values are reduced. After the second week, the GCT fibers affected by infarction had higher MD values than the CGCT. The MD gradually increased and was equal to the MD of the CSF after the eighth week. During that time, the cellular structure disappeared when the swollen cells ruptured. The extracellular space and the water content of the tissues increased. The diffusion resistance decreased. The MD increased and was eventually equal to the MD of the CSF. This outcome suggests necrosis and tissue liquefaction.

There were no differences between the FA and MD of the UGCT and CGCT during the first 3 weeks. After the fourth week, the FA gradually decreased, while the MD gradually increased (Table). However, the ranges were lower compared with those of the GCT fibers affected by infarction. The degeneration and atrophy of the UGCT, including the disintegration, demyelination, and increased water content in the tissues, are a delayed minimal process compared with the GCT fibers affected by infarction. This suggests several approaches regarding the intervention and treatment of UGCT. First, the initial 3 weeks poststroke are a key phase for interventions because significant damage has not yet occurred. The degeneration of UGCT may be delayed or alleviated if effective interventions have been performed. Second, the FA and MD of the UGCT were not stabilized until 1 year after the infarction. Because the demyelination and degeneration of fibers in the unaffected GCT are chronic and continuous, the interventions and treatments for UGCT may last for a longer time. Third, additional research should focus on studying UGCT in the long term. Changes in the FA and MD may suggest possible modifications or plasticity of the fibers.

Diffusion indices may be valid markers of GCT integrity after ischemic stroke and predictors of vision prognosis. ${ }^{20-23}$ For example, MR tractography predicts visual field defects following temporal lobe resection. ${ }^{19}$ Vision recovery after perinatal stroke as evidenced by functional and diffusion MR imaging has also been shown. ${ }^{31}$ White matter reorganization is closely related to the status of axons, including axonal attenuation. The MR imaging diffusion entropy method primarily depends on axonal attenuation rather than axonal orientation and is a potentially useful measurement for detecting white matter reorganization. ${ }^{1,32}$ These techniques assist in investigating complex crossing fibers and white matter plasticity, which contributes to our ultimate goal of vision recovery after ischemic stroke.

\section{CONCLUSIONS}

In this study, we explored changes in GCT fiber integrity by investigating the mean FA and MD from 1 week until $>1$ year after stroke onset. Diffusion indices provide information on the underlying microanatomic or pathologic changes in white matter fiber bundles. ${ }^{25,26,33,34}$ The GCT fibers affected by infarction and UGCT undergo different processes of disintegration. The GCT fibers affected by infarction disintegrated from stroke onset until 
the eighth week poststroke. The UGCT did not disintegrate until the fourth week poststroke, though to a lesser degree than the GCT fibers affected by infarction. DTI-derived measures are valid markers of geniculocalcarine tract integrity after ischemic stroke.

\section{REFERENCES}

1. Mohr JP, Foulkes MA, Polis AT, et al. Infarct topography and hemiparesis profiles with cerebral convexity infarction: the Stroke Data Bank. J Neurol Neurosurg Psychiatry 1993;56:344-51

2. Saver JL, Johnston KC, Homer D, et al. Infarct volume as a surrogate or auxiliary outcome measure in ischemic stroke clinical trials. Stroke 1999;30:293-98

3. Binkofski F, Seitz RJ, Arnold S, et al. Thalamic metabolism and corticospinal tract integrity determine motor recovery in stroke. Ann Neurol 1996;39:460-70

4. Crafton KR, Mark AN, Cramer SC. Improved understanding of cortical injury by incorporating measures of functional anatomy. Brain 2003;126:1650-59

5. Schiemanck SK, Kwakkel G, Post MW, et al. Impact of internal capsule lesions on outcome of motor hand function at one year poststroke. J Rehabil Med 2008;40:96-101

6. Shelton FN, Reding MJ. Effect of lesion location on upper limb motor recovery after stroke. Stroke 2001;32:107-12

7. Ciccarelli O, Catani M, Johansen-Berg H, et al. Diffusion-based tractography in neurological disorders: concepts, applications, and future developments. Lancet Neurol 2008;7:715-27

8. Lindenberg R, Renga V, Zhu LL, et al. Structural integrity of corticospinal motor fibers predicts motor impairment in chronic stroke. Neurology 2010;74:280-87

9. Zhu LL, Lindenberg R, Michael P, et al. Lesion load of the corticospinal tract predicts motor impairment in chronic stroke. Stroke 2010;41:910-15

10. Jang SH, Bai D, Son SM, et al. Motor outcome prediction using diffusion tensor tractography in pontine infarct. Ann Neurol 2008;64:460-65

11. Kunimatsu A, Aoki S, Masutani Y, et al. Three-dimensional white matter tractography by diffusion tensor imaging in ischaemic stroke involving the corticospinal tract. Neuroradiology 2003; 45:532-35

12. Yamada $\mathrm{K}$, Mori S, Nakamura $\mathrm{H}$, et al. Fiber tracking method reveals sensor motor pathway involvement in stroke patients. Stroke 2003;34:E159-62

13. Konishi J, Yamada K, Kizu O, et al. MR tractography for the evaluation of the functional recovery from lenticulostriate infarcts. $\mathrm{Neu}$ rology 2005;64:108-13

14. Nelles M, Gieseke J, Flacke S, et al. Diffusion tensor pyramidal tractography in patients with anterior choroidal artery infarcts. AJNR Am J Neuroradiol 2008;29:488-93

15. Kunimatsu A, Itoh D, Nakata $Y$, et al. Utilization of diffusion tensor tractography in combination with spatial normalization to assess involvement of the corticospinal tract in capsular/pericapsular stroke. J Magn Reson Imaging 2007;26:1399-404

16. Puig J, Pedraza S, Blasco G, et al. Acute damage to the posterior limb of the internal capsule on diffusion tensor tractography as an early imaging predictor of motor outcome after stroke. AJNR Am J Neuroradiol 2011;32:857-63

17. Radlinska B, Ghinani S, Leppert IR, et al. Diffusion tensor imaging, permanent pyramidal tract damage, and outcome in subcortical stroke. Neurology 2010;75:1048-54

18. Schaechter JD, Fricker ZP, Perdue KL, et al. Microstructural status of ipsilesional and contralesional corticospinal tract correlates with motor skill in chronic stroke patients. Hum Brain Mapp 2009;30: 3461-74

19. Seghier ML, Lazeyras F, Zimine S, et al. Visual recovery after perinatal stroke evidenced by functional and diffusion MRI: case report. BMC Neurol 2005:26;5:17

20. Wieshmann UC, Symms MR, Clark CA, et al. Wallerian degeneration in the optic radiation after temporal lobectomy demonstrated in vivo with diffusion tensor imaging. Epilepsia 1999;40:1155-58

21. Werring DJ, Clark CA, Parker GJ, et al. A direct demonstration of both structure and function in the visual system: combining diffusion tensor imaging with functional magnetic resonance imaging. Neuroimage 1999;9:352-61

22. Yamamoto A, Miki Y, Urayama S, et al. Diffusion tensor fiber tractography of the optic radiation: analysis with 6-, 12-, 40-, and 81directional motion-probing gradients, a preliminary study. AJNR Am J Neuroradiol 2007;28:92-96

23. Yamamoto T, Yamada K, Nishimura T, et al. Tractography to depict three layers of visual field trajectories to the calcarine gyri. Am J Ophthalmol 2005;140:781-85

24. Miller NR. Diffusion tensor imaging of the visual sensory pathway: are we there yet? Am J Ophthalmol 2005;140:896-97

25. Wan SH, Zhang XL, Xie XL. The initial clinical application of MR- DTI and DTT in the adult optic radiation. J Clin Radiol 2007; 26:1076-79

26. Tong Y. Neuro-ophthalmology. In: Ge J, ed. Ophthalmology. Beijing: People's Medical Publishing House; 2005:323-25

27. Zeng LF. Visual pathway and visual center. In: Li FM, ed. Chinese Ophthalmology. Vol 1. 2nd ed. Beijing: People's Medical Publishing House; 2005:180-90

28. Dong Q. Atherosclerosis cerebral infarction. In: Chen JZ, ed. Practice of Internal Medicine. Vol 2. 12th ed. Beijing: People's Medical Publishing House; 2007:2669-74

29. Le Bihan D. Looking into the functional architecture of the brain with diffusion MRI. Nat Rev Neurosci 2003;4:469-80

30. Pierpaoli C, Basser PJ. Towards a quantitative assessment of diffusion anisotropy. Magn Reson Med 1996;36:893-906

31. Powell HW, Parker GJ, Alexander DC, et al. MR tractography predicts visual field defects following temporal lobe resection. Neurology 2005;65:596-99

32. Jiang Q, Zhang ZG, Chopp M. MRI evaluation of white matter recovery after brain injury. Stroke 2010;41:S112-13

33. Chanraud S, Zahr N, Sullivan EV, et al. MR diffusion tensor imaging: a window into white matter integrity of the working brain. Neuropsychol Rev 2010;20:209-25

34. Parekh MB, Carney PR, Sepulveda H, et al. Early MR diffusion and relaxation changes in the parahippocampal gyrus precede the onset of spontaneous seizures in an animal model of chronic limbic epilepsy. Exp Neurol 2010;224:258-70 